ARTICLE

Received 30 Jan 2014 | Accepted 31 Mar 2014 | Published 8 May $2014 \quad$ DOl: 10.1038/ncomms4772

\title{
A Krüppel-like factor downstream of the E3 ligase WWP-1 mediates dietary-restriction-induced longevity in Caenorhabditis elegans
}

\author{
Andrea C. Carrano ${ }^{1, \dagger}$, Andrew Dillin ${ }^{1,2} \&$ Tony Hunter ${ }^{1}$
}

The HECT ubiquitin E3 ligase WWP-1 is a positive regulator of lifespan in response to dietary restriction (DR) in Caenorhabditis elegans. However, substrates of WWP-1 for ubiquitylation in the DR pathway have not yet been identified. Here we identify the $C$. elegans Krüppel-like factor, KLF-1, as an essential and specific regulator of DR-induced longevity and a substrate for ubiquitylation by WWP-1. Knockdown of klf-1 suppresses the extended lifespan of both DR animals and wwp-1-overexpressing animals, indicating that KLF-1 functions within the same pathway as WWP-1. In addition, overexpression of klf-1 in the intestine is sufficient to extend the lifespan of WT animals on an ad libitum diet, and requires wwp-1 or pha-4/FoxA. We demonstrate that WWP-1 directly interacts with KLF-1 and mediates multiple monoubiquitylation of KLF-1 in vitro and in cellulo. Our data support a model in which modulation of KLF-1 by WWP-1 regulates diet-restriction-induced longevity.

\footnotetext{
${ }^{1}$ Molecular and Cell Biology Laboratory, The Salk Institute for Biological Studies, La Jolla, California 92037, USA. ${ }^{2}$ The Howard Hughes Medical Institute, University of California, Berkeley, California 94720, USA. †Present address: Department of Pediatrics, University of California, San Diego, La Jolla, California 92093, USA. Correspondence and requests for materials should be addressed to T.H. (email: hunter@salk.edu).
} 
T he Krüppel-like factor (KLF) family of gene regulatory proteins are transcription factors implicated in many biological processes that include proliferation, differentiation, growth, development, survival and responses to external stress $^{1}$. The characteristic feature of KLF family members is the presence of three Krüppel-like zinc fingers at the carboxy (C)terminus, which bind to CACCC elements and GC-rich regions of DNA, to mediate activation and/or repression of transcription. In addition, KLF proteins have nuclear localization sequences, which occur immediately adjacent to, or within, the zinc-finger motifs. The amino $(\mathrm{N})$-terminal regions of KLF proteins are much more variable, allowing the interaction with different coactivators, corepressors and modifiers, resulting in functional diversity and specificity.

KLF proteins are present in species ranging from Caenorhabditis elegans to humans, with humans having at least 17 members. Three KLFs are predicted from the C. elegans genome: klf- 1 (F56F11.3), klf-2 (F53F8.1) and klf-3 (F54H5.4). All three KLFs share the highest sequence identity with members of mammalian KLFs in the C-terminal $\mathrm{C}_{2} \mathrm{H}_{2}$ domains but little homology in their N-terminal regions. Roles for klf-1 in fat regulation, germline cell death and phagocytosis have been described ${ }^{2,3}$. klf-3 (also known as mua-1) has been implicated in fat metabolism and cell matrix attachment ${ }^{4-7}$.

KLF members participate in regulation of gene transcription through the interaction with intracellular signalling molecules and transcriptional cofactors. In addition, coregulatory proteins modify KLF family members via acetylation, phosphorylation, ubiquitylation and sumoylation to refine their transcriptional activity $^{1}$. These post-translational modifications are thought, at least in part, to affect KLF family interactions with transcriptional cofactors, thus mediating the differential biological effects of KLFs. The mammalian family of WW domain-containing HECT E3 ligases has been shown to promote conjugation of ubiquitin to several KLF family members resulting in different protein fates. Mammalian WWP1 has been shown to mediate ubiquitylation of both KLF2 and KLF5, targeting these proteins for degradation ${ }^{8-10}$. Itch promotes monoubiquitylation of TIEG1/KLF10 and enhances its transcriptional activation of the Foxp3 promoter and Foxp3 expression ${ }^{11}$. Itch has also been shown to target phosphorylated TIEG1/KLF10 for K27-linked polyubiquitylation and prevent its nuclear translocation ${ }^{12}$.

We previously identified the C. elegans WWP-1 E3 ligase and its associated E2 ubiquitin-conjugating enzyme, UBC-18, as positive regulators of lifespan in response to DR ${ }^{13}$. WWP1 is an E3 ubiquitin ligase orthologous to human WWP1, WWP2 and Itch. In addition to their C-terminal catalytic HECT domain, these ligases have an $\mathrm{N}$-terminal $\mathrm{Ca}^{2+} /$ lipid-binding $\mathrm{C} 2$ domain and four WW domains, modular protein-interaction domains that recognize short proline motifs in target proteins. While the ubiquitin ligase activity of WWP-1 is essential for DR-induced longevity, a substrate for WWP-1 to modulate this response is still unknown. Here we demonstrate that a C. elegans KLF, KLF-1, is a substrate for ubiquitylation by WWP-1. Like $w w p-1, k l f-1$ is essential and specific for the longevity response to decreased nutrient intake. In addition, genetic analysis reveals that $k l f-1$ and $w w p-1$ function together within the intestine to modulate longevity in response to DR.

\section{Results}

klf-1 is required for the extension of lifespan by DR. Loss of wwp-1 function suppresses the extended longevity of eat-2 mutant animals, a genetic model for DR, and other forms of diet restriction ${ }^{13}$. To identify potential substrates of WWP-1 that regulate DR-induced longevity, we performed a targeted RNAi screen by selecting worm orthologues of known ubiquitylated substrates of mammalian WWP-1 family members (WWP-1, WWP2 and Itch). None of the 12 genes we analysed were able to extend lifespan in wild-type (N2) worms when knocked down (Fig. 1 and Supplementary Table 1). However, we found that loss of one gene, klf-1, completely suppressed the extended longevity of eat-2 mutant animals but did not affect N2 lifespan (Fig. 2a and b, Supplementary Fig. 1a and b and Supplementary Tables 2 and 3). This suppression appears to be exerted during adulthood, as we still observed this effect when we initiated RNAi at day 1 of adulthood (Fig. 2a). Previous studies have found that klf-1 knockdown affects egg-laying behaviour ${ }^{3}$. To rule out the possibility that the reduced lifespan noted on loss of $k l f-1$ is due to premature death caused by egg-laying defects, we repeated the lifespans in a temperature-sensitive sterile background (eat-2(ad1116);CF512:fer-15(b26);fem-1(hc17)) and still observed complete suppression of lifespan with loss of klf-1 (Supplementary Fig. 1c and Supplementary Table 3). The suppression of DR-extended lifespan by $k l f-1$ depletion is unlikely to be due to increased food intake because no difference in pharyngeal pumping rates with knockdown of $k l f-1$ in $\mathrm{N} 2$ or eat-2(ad1116) worms was observed (Supplementary Table 4).

We also tested whether the loss of klf-1 suppressed the extended longevity of animals diet restricted by reduced food intake imposed by bacterial dilution in liquid culture ${ }^{13,14}$. Ideally we wanted to use a $k l f-1$-null allele for this experiment, because RNAi depletion cannot be achieved in liquid culture. However, the only two mutant alleles of klf-1, tm731 and tm1110, do not appear to be null alleles as both have only short intronic sequence deletions and have a wild-type phenotype ${ }^{3}$ (Supplementary Fig. 2 and Supplementary Table 3), and, in consequence, we knocked down expression of klf-1 by RNAi from hatching (L1) to day 3 adults before moving them to liquid cultures of OP50 (Fig. 2c). Control N2 animals grown under DR conditions had a lifespan of nearly double that of animals fed ad libitum (Fig. 2d). Loss of klf-1 did not affect lifespan of animals fed ad libitum. In contrast, the lifespan of klf-1-depleted animals undergoing DR was significantly shorter when compared with control animals (Fig. 2d and Supplementary Table 2). As these animals were fed bacteria expressing double-stranded RNA (dsRNA) for only the first 5 days of life, temporary RNAi knockdown of klf-1 in these experiments may explain why we did not observe complete suppression of longevity in animals undergoing DR. Indeed,

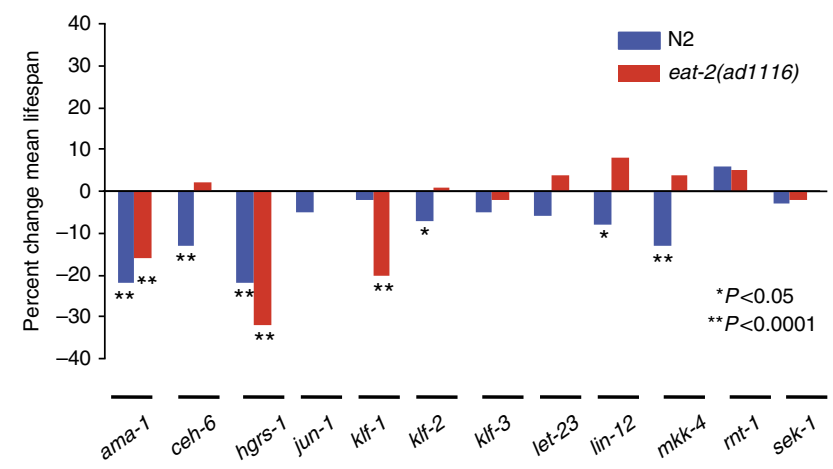

Figure 1 | Targeted RNAi screen to identify WWP-1 substrates involved in lifespan regulation. Percent change in mean lifespan in N2 worms (blue) or eat-2(ad1116) worms (red) treated with RNAi to the indicated genes only during adulthood compared with empty vector. Mean percent change of 1-4 independent experiments is shown. ${ }^{\star} P<0.05$, ${ }^{\star \star} P<0.0001$ (compared with same strain worms grown on HT115 bacteria harbouring the RNAi plasmid vector; Mantel-Cox log-rank test). Lifespan values are given in Supplementary Table 1. 
a

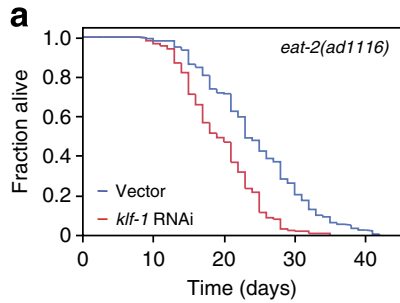

C

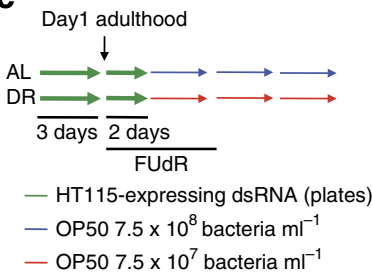

e

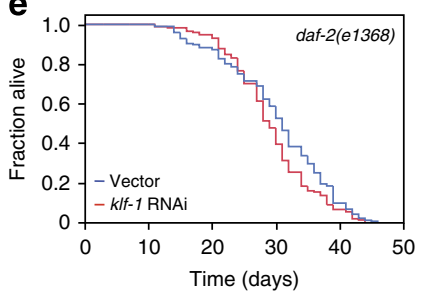

b

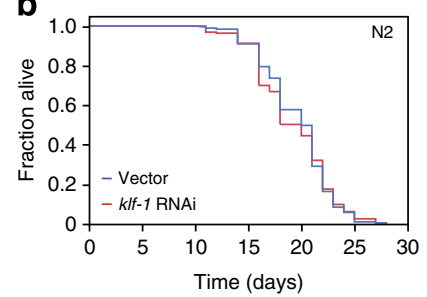

d
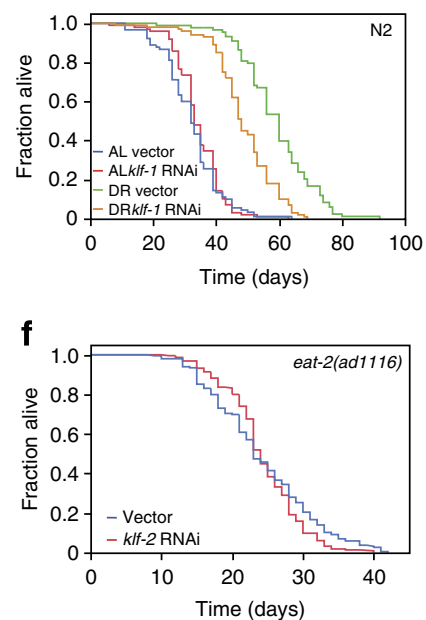

g

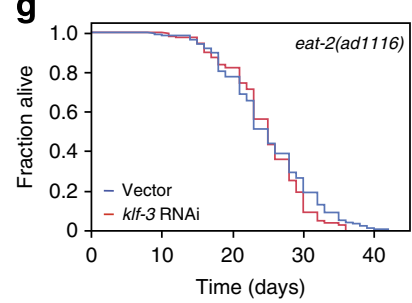

Figure 2 | klf-1 is required and specific for the extension of lifespan by dietary restriction. Lifespan curves represent combined data from multiple independent experiments. Lifespan values are given in Supplementary Table 2. (a,b) Lifespan analysis of eat-2(ad1116) mutant worms (a) or N2 worms (b) fed bacteria expressing kIf-1 dsRNA only during adulthood or control vector. (c) Schematic representation of liquid culture bacterial dilution experiments with RNAi. (d) Lifespan analysis of N2 worms grown in diet restricted (DR) or ad libitum (AL) E. coli (OP50) concentrations in liquid culture from day 3 adulthood after feeding bacteria expressing klf-1 dsRNA or control vector on plates. (e) Lifespan analysis of daf-2(e1368) mutant animals fed klf-1 dsRNA only during adulthood or control vector. RNAi depletion of klf-2 or klf-3 during adulthood had no effect on daf-2(e1368) lifespan (data not shown). (f,g) Lifespan analysis of eat-2(ad1116) mutant worms fed bacteria expressing klf-2 (f), klf-3 (g) dsRNA only during adulthood or control vector.

under identical conditions, we observed a partial suppression of DR lifespan with $w w p-1$ knockdown, while use of a $w w p-1$-null allele gave a much stronger effect (Supplementary Fig. 3, Supplementary Table 3 and ref. 13). These results indicate that reduction of $k l f-1$ expression early in life is sufficient to reduce the extended longevity of DR animals.

To determine whether $k l f-1$ was acting specifically to affect the DR pathway, we examined its effect on other pathways that influence longevity. Reduced IIS, by mutation of the insulin/IGF1 receptor, daf-2, increases longevity. RNAi depletion of $k l f-1$, like $w w p-1$, had no effect on the long lifespan of insulin/IGF-1 receptor (daf-2) mutants (Fig. 2e and Supplementary Table 2). In addition, we tested whether $k l f-1$ was required for the long lifespan of animals with reduced mitochondrial electron transport chain activity. We observed suppressive effects on lifespan extension in long-lived isp-1 mitochondrial mutant animals with $k l f-1$ knockdown. However, this was not specific for $k l f-1$, as RNAi-mediated reduction of $k l f-2$ and $k l f-3$, the two other KLFs in C. elegans, also gave similar minor lifespan reductions (Supplementary Fig. 4 and Supplementary Table 3). In addition, lifespan modulation directed by $k l f-1$ is specific, as RNAi of $k l f-2$ and $k l f-3$ did not reduce the lifespan of eat- 2 mutant animals (Fig. $2 \mathrm{f}$ and $\mathrm{g}$ and Supplementary Table 2). In sum, $k l f-1$ has a specific and essential role in regulating the response to nutrient intake and longevity.

$w w p-1$ and $k l f-1$ function together to regulate longevity. $k l f-1$ is primarily expressed in the intestine of the worm ${ }^{3}$, a site where longevity cues are expressed ${ }^{15}$. We asked if $k l f-1$ functions in this tissue to regulate DR-induced longevity. sid-1 encodes a transmembrane protein required for transporting RNAi silencing signals between cells. Loss of sid-1 does not allow dsRNA to be distributed into peripheral cells ${ }^{16}$. Indeed, when eat-2(ad1116);sid-1(qt9) animals were fed bacteria expressing klf-1 dsRNA, we no longer observed a reduction in lifespan (Fig. 3a and Supplementary Table 2), presumably because the $k l f-1$ dsRNA could not enter the intestinal cells. Lifespans were then performed with eat-2(ad1116);sid-1(qt9) mutant animals in which sid-1 was restored by tissue-specific expression of sid-1 cDNA. sid-1 was rescued in transgenic lines under the control of the $g l y$-19 intestine-expressing promoter or the myo-3 body wall muscle-expressing promoter. Restoring sid-1 in the intestine and feeding $k l f-1$ dsRNA-producing bacteria to deplete $k l f-1$ selectively in the intestine led to complete suppression of longlived eat-2 mutant lifespan (Fig. $3 \mathrm{~b}$ and Supplementary Table 2). In contrast, muscle-specific knockdown of klf- 1 had no significant effect on lifespan (Fig. 3c and Supplementary Table 2). WWP-1 is also expressed in the intestine ${ }^{13}$. Like klf-1, knockdown of $w w p-1$ specifically in the intestine, but not muscle, led to suppression of long-lived eat-2 mutant lifespan (Fig. $3 \mathrm{~b}$ and $\mathrm{c}$ and Supplementary Table 2). Taken together, a major site of action of WWP-1 and KLF-1 to regulate the response to DR appears to be the intestine.

Because loss of $k l f-1$ suppressed longevity, we asked if increased expression of $k l f-1$ was sufficient to increase longevity. We were unsuccessful in generating stable $k l f-1$ transgenic lines by microinjection of a plasmid containing the entire coding sequence 
a

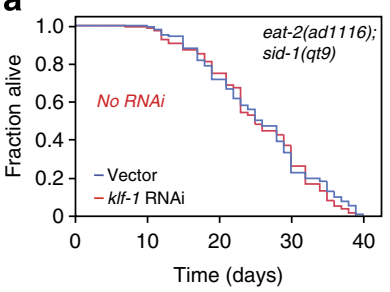

b

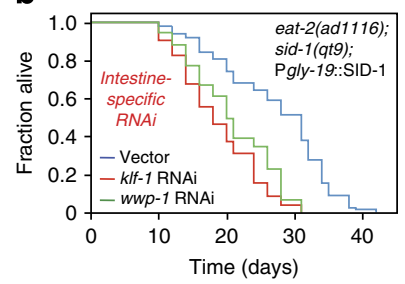

C

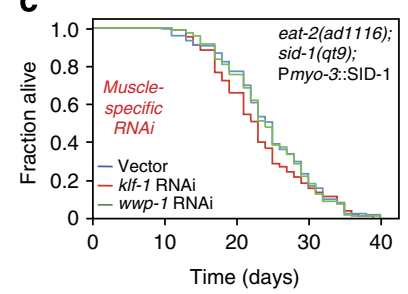

Figure 3 | The intestine is a major site of action of wwp-1 and klf-1 to regulate the dietary restriction response. (a) Lifespan analysis of eat-2(ad1116); sid-1(qt9) mutant worms fed bacteria expressing dsRNA to klf-1 or control vector. (b,c) Lifespan analysis of eat-2(ad1116);sid-1(qt9) worms with restored SID-1 expression in the intestines (b) or muscle tissues (c). Worms were fed bacteria expressing dsRNA to kIf-1, wwp-1 or control vector.

a

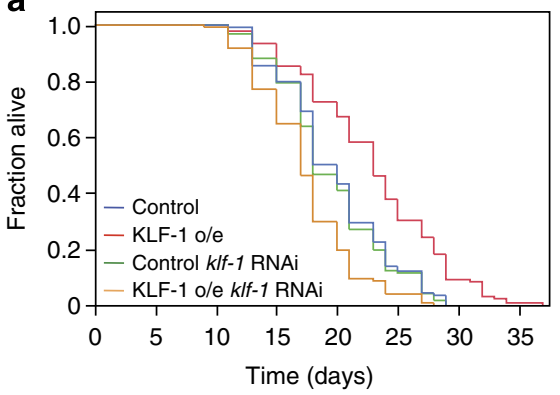

C

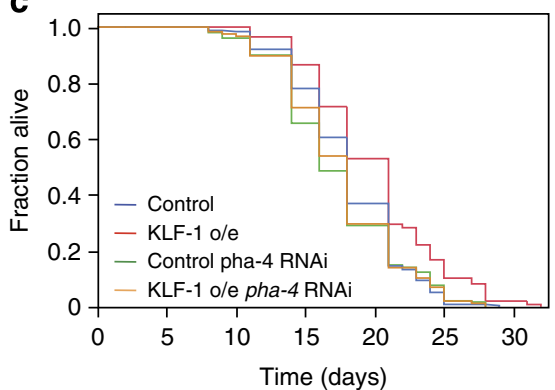

b

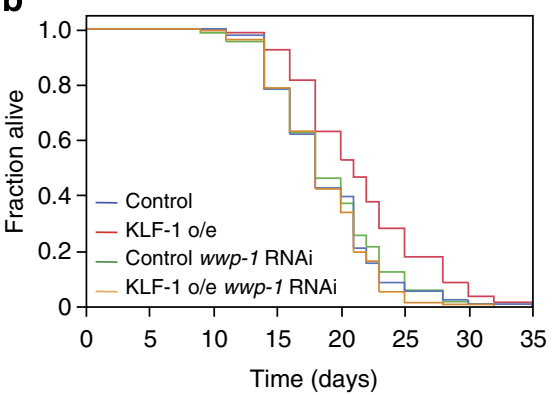

d

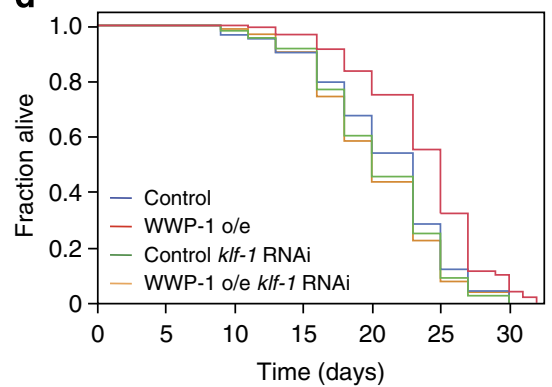

Figure 4 | wwp-1 and klf-1 function together to regulate diet-restriction-induced longevity. (a-c) Lifespan analysis of worms overexpressing KLF-1 in the intestine (KLF-1 o/e) or control worms fed bacteria expressing klf-1 (a), wwp-1 (b) or pha-4 (c) dsRNA from hatching (L1) or control vector. (d) Lifespan analysis of WWP-1-overexpressing worms (WWP-1 o/e) or control worms fed bacteria expressing klf-1 dsRNA from hatching (L1) or control vector.

of $k l f-1$ and $2 \mathrm{kbp}$ of $5^{\prime}$ upstream sequence from its ATG at low concentrations. Several possibilities could account for our inability to achieve overexpression of $k l f-1$, including expression levels as well as spatial control of expression. For this reason, we tested whether spatially controlled ectopic expression of $k l f-1$ could influence longevity by generating a transgenic line carrying an intestine-specific construct that expressed $k l f-1 \mathrm{cDNA}$ fused to a C-terminal tdTomato fluorescent protein. We found that tdTomato expression was very low in this line compared with control lines that express tdTomato alone under the same promoter (Supplementary Fig. 5a), suggesting that KLF-1 overexpression may be toxic. Nevertheless, KLF-1 was specifically expressed, as the tdTomato fluorescent signal was reduced on klf-1 dsRNA treatment, but not control vector (Supplementary Fig. 5b). We found that this modest level of overexpression of $k l f-1$ in worms extends lifespan by approximately $15 \%$, and this extension was reverted with klf-1 dsRNA treatment (Fig. 4a and Supplementary Table 2). This extension is similar to that we find with $w w p-1$ overexpression (Fig. $4 \mathrm{~d}$, Supplementary Table 2 and ref. 13). The increase in longevity induced by klf-1 expression was suppressed when $w w p-1$ expression was reduced by RNAi, indicating that the ability of $k l f-1$ to extend lifespan requires $w w p-1$ (Fig. $4 \mathrm{~b}$ and Supplementary Table 2). Knockdown of pha-4, the FoxA transcription factor required for DR-induced longevity ${ }^{14}$, also suppressed the extended lifespan of klf-1overexpressing animals (Fig. 4c and Supplementary Table 2) placing $k l f-1$ in the same pathway as pha-4.

Increased expression of $w w p-1$ extends the lifespan of $\mathrm{N} 2$ worms ${ }^{13}$ (Fig. $4 \mathrm{~d}$ and Supplementary Table 2). Stable transgenic lines that express an N-terminal green fluorescent proteinWWP-1 fusion protein, under the control of the endogenous $w w p-1$ promoter live longer than control animals ${ }^{13}$. Knockdown of $k l f-1$ suppressed the extended lifespan of $w w p$-1-overexpressing animals (Fig. 4d and Supplementary Table 2), indicating that $w w p-1$ requires $k l f-1$ to extend longevity and that $k l f-1$ functions in the same pathway as $w w p-1$ to regulate longevity in response to diet.

WWP-1 associates with and ubiquitylates KLF-1. The ubiquitin ligase activity of WWP-1 is essential for DR-induced longevity, indicating that WWP-1 functions to ubiquitylate a substrate(s) to mediate its longevity effects ${ }^{13}$. Our genetic analysis of klf-1 suggests that WWP-1 may target KLF-1 for ubiquitylation. Based 
on these data, we first investigated whether WWP-1 could directly interact with KLF-1. On co-expression of FLAG-tagged KLF-1 and hemagglutinin protein (HA)-tagged WWP-1 in human HEK293T cells, WWP-1 was co-immunoprecipitated with KLF-1 (Fig. 5a). To determine if this interaction is direct, we performed pull-down studies using GST-WWP-1 fusion proteins and ${ }^{35}$ S-labelled in vitro-translated KLF-1 and found that GST-WWP-1 could pull down KLF-1, whereas GST protein alone did not (Fig. 5b, left panel). In contrast, the transcription factor PHA-4 did not bind to WWP-1 under the same conditions (Fig. 5b, right panel). A GST fusion protein containing only the WW domains of WWP-1 (aa 215-412) was sufficient to bind KLF-1, showing that the WW domains of WWP-1 mediate the interaction between KLF-1 and WWP-1 (Fig. 5c). These results indicate that WWP-1 can form a complex with KLF-1.

To determine if the interaction between these two proteins results in ubiquitylation of KLF-1 in vivo, we transfected HEK293T cells with HA-tagged ubiquitin, FLAG-KLF-1 and HA-WWP-1. Immunoblot analysis of immunoprecipitated FLAG-KLF-1 from the denatured extracts with an ubiquitin antibody revealed a ladder of bands representing ubiquitylated KLF-1 that was absent when HA-WWP-1 was not expressed or with expression of a catalytically inactive WWP-1 mutant (C762A) (Fig. 5d). The ability of WWP-1 to promote ubiquitylation of KLF-1 was confirmed by performing an in vitro

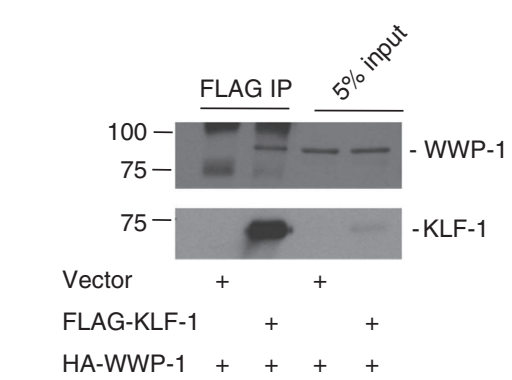

d

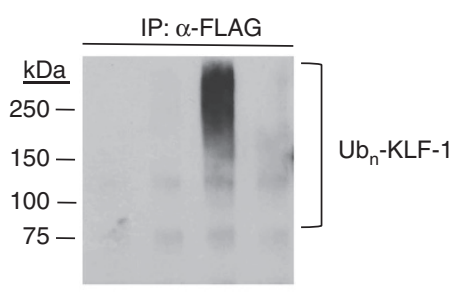

IB: $\alpha-U b$

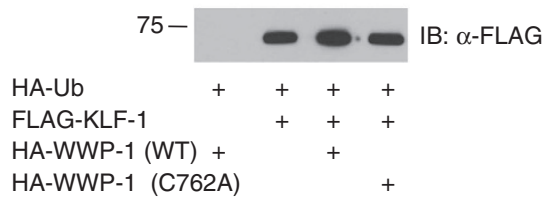

ubiquitylation assay using bacterially expressed E1, UBC-18 (E2) and WWP-1 (E3) and ${ }^{35}$ S-labelled in vitro-translated KLF-1 substrate. On addition of WWP-1, we observed a ladder of more slowly migrating bands of KLF-1 that was not observed when we used the catalytically inactive WWP-1(C762A) mutant protein (Fig. 5e). This modification is ubiquitylation, as its presence was dependent on the addition of recombinant ubiquitin.

KLF-1 is preferentially monoubiquitylated by WWP-1. As various types of ubiquitin modification are linked to distinct physiological functions, we investigated the type of ubiquitylation occurring on KLF-1 mediated by WWP-1. Ubiquitin can be covalently conjugated to substrates in several ways. A single ubiquitin can be conjugated to a single site (monoubiquitylation) or multiple sites (multiple monoubiquitylation), or multiple ubiquitins can be linked via one of the seven lysine residues on ubiquitin to form polyubiquitin chains (polyubiquitylation). K48linkages are abundant, being the predominant signal for proteins destined for degradation by the proteasome. Immunoblot analysis of immunoprecipitated FLAG-KLF-1 from HEK293T cells with an antibody that specifically recognizes K48-linked polyubiquitin chains revealed no difference in the level of ubiquitylated species when KLF-1 was expressed with WWP-1 compared with vector or WWP-1 (C762A) mutant (Fig. 6a, left panel). We detected a

b

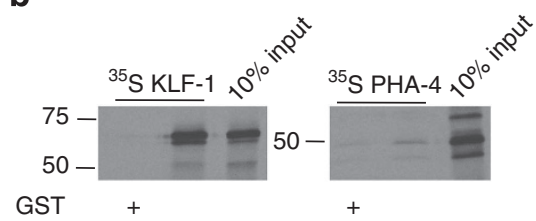

$$
\text { GST WWP-1 + }+
$$

C

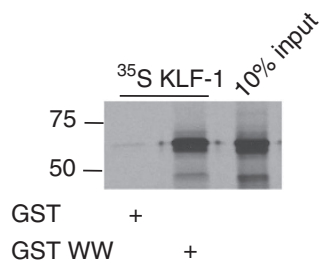

e

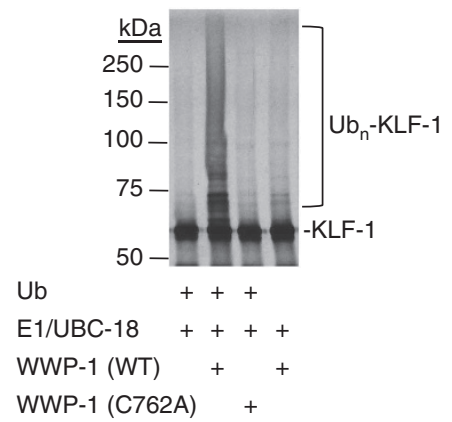

Figure 5 | WWP-1 associates with and ubiquitylates KLF-1. (a) HA-WWP-1 and FLAG-KLF-1 were expressed in HEK293T cells. Lysates were immunoprecipitated with FLAG antibodies and probed with antibodies against HA (top panel) or FLAG (bottom panel). Molecular size in kilodaltons (kDa) is indicated on the left. (b) GST pull-down assay in which GST-WWP-1 or GST alone bound to glutathione beads was incubated with in vitro-translated ${ }^{35}$ S-labelled KLF-1 (left panel) or PHA-4 (right panel). (c) GST pull-down with ${ }^{35}$ S-labelled KLF-1 and a GST fusion protein containing only the WW domains of WWP-1. (d) In vivo ubiquitylation of KLF-1 by WWP-1. HEK293T cells were transfected with HA-tagged ubiquitin and FLAG-KLF-1 and/or HA-WWP-1. Denatured lysates were immunoprecipitated with anti-FLAG beads and analysed by immunoblot with an ubiquitin antibody. Similar banding pattern was seen when probed with an anti-HA antibody (data not shown). Bottom panel shows that similar amounts of KLF- 1 were immunoprecipitated. (e) In vitro ubiquitylation assay using ${ }^{35}$ S-labelled KLF-1 incubated with bacterially expressed recombinant E1, UBC-18 and GST-WWP-1. Recombinant ubiquitin was added to the reaction where indicated. Uncropped images from the autoradiography scans and immunoblots are shown in Supplementary Fig. 9. 
a

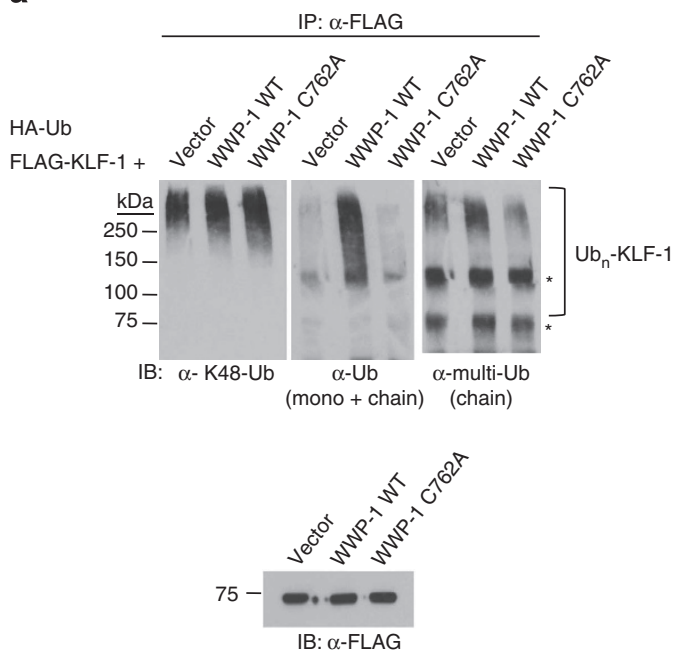

b

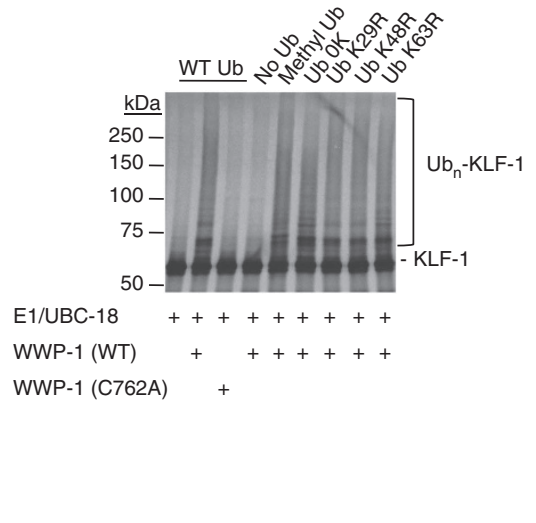

Figure 6 | KLF-1 is preferentially monoubiquitylated by WWP-1. (a) In vivo ubiquitylation assay similar to Fig. $5 \mathrm{~d}$ except $0.1 \mathrm{mM}$ Bortezomib was added to the cells. Denatured lysates were immunoprecipitated with anti-FLAG beads and analysed by immunoblot with the indicated ubiquitin antibodies. The asterisks $\left(^{*}\right)$ indicate nonspecific bands present in all samples. Bottom panel shows that similar amounts of KLF-1 were immunoprecipitated. (b) In vitro ubiquitylation assay using ${ }^{35}$ S-labelled KLF-1 incubated with bacterially expressed recombinant E1, UBC-18 and GST-WWP-1. Recombinant WT ubiquitin or the indicated ubiquitin mutants were added to the reaction where indicated. Uncropped images from the autoradiography scan and immunoblots are shown in Supplementary Fig. 10.

slight increase in KLF-1 high-molecular weight bands with WWP-1 WT expression when the same experiment was probed with an antibody that specifically recognizes polyubiquitylated substrates but not monoubiquitylated substrates (Fig. 6a, right panel). In contrast, we saw a significantly greater increase of ubiquitylated bands with an antibody that recognizes both polyubiquitylated and monoubiquitylated species (Fig. 6a, middle panel), indicating that these bands represent KLF-1 conjugated with a ubiquitin monomer at multiple sites.

To further address whether the observed pattern represented polyubiquitylation or multiple monoubiquitylation, various ubiquitin mutants were analysed in our in vitro assay. The use of methylated ubiquitin, which prevents formation of polyubiquitin chains via its lysine residues, or a recombinant ubiquitin, in which all seven lysine residues of ubiquitin were mutated to arginines (K0), generated a pattern similar to WT ubiquitin. Analysis of single lysine ubiquitin mutants, K29R, K48R and K63R also resulted in a similar banding pattern (Fig. 6b). Therefore, it appears that the banding pattern found in our in vitro assay resembles multiple monoubiquitylation of KLF-1 by WWP-1. Taken together, our findings suggest that WWP-1 preferentially catalyses attachment of mono- rather than polyubiquitin moieties to KLF-1.

\section{Discussion}

Our study identifies the C. elegans KLF, KLF-1 as a substrate for ubiquitylation by WWP-1. Mammalian orthologues of WWP-1 have been shown to target multiple transcription factors for proteasome-dependent degradation including KLF5 (refs 9,10), KLF2 (ref. 8), Oct-4 (ref. 17), Runx2 (ref. 18), p63 (ref. 19), p73 (ref. 20), JunB and cJun ${ }^{21}$. Ubiquitin modifications mediated by mammalian WWP-1 orthologues that do not lead to proteasomedependent proteolysis have also been found to regulate transcription factor activity. For example, WWP1 increases the transcriptional activity of a p63 isoform $(\Delta \mathrm{N} 63)$ through generation of Lys63-linked polyubiquitin chains ${ }^{22}$. WWP2 monoubiquitylates the homeodomain transcription factor
Goosecoid (Gsc), which augments its transcriptional activity ${ }^{23}$ Monoubiquitylation of TIEG1/KLF10 by Itch has been shown to directly regulate KLF10 transcriptional activity, while K27-linked ubiquitylation by the same E3 modulates its cellular localization (described above) $)^{11,12}$. In addition, Itch suppresses the transactivation activity of p45/NF-E2 by the addition of a K63linked chain causing it to be retained in the cytoplasm ${ }^{24}$, while monoubiquitylation of $\mathrm{p} 53$ by WWP1 negatively regulates its transcriptional activity by shuttling it to the cytoplasm ${ }^{25}$.

Our genetic data indicate that $k l f-1$, like $w w p-1$, is a positive regulator of lifespan suggesting that KLF-1 activity is enhanced on DR. Our data support a model in which ubiquitylation of KLF-1 by WWP-1 regulates the DR-longevity response. While we were able to demonstrate WWP-1-dependent ubiquitylation of KLF-1 both in vitro and in cellulo, we were not able to detect ubiquitylation of KLF-1 in the worm. This may be a detection problem due to the low expression of tagged KLF-1 in our transgenic animals. However, our results using these two systems indicate that WWP-1 mediates multiple monoubiquitylation of KLF-1. As monoubiquitylation does not signal proteasomemediated degradation, KLF-1 modification by WWP-1 should not affect its stability. Indeed, we did not observe discernable differences in tdTomato fluorescence when $w w p-1$ was knocked down in worms that express KLF-1-tdTomato fusion protein in the intestine (Supplementary Fig. 6).

It is unclear how WWP-1-mediated ubiquitylation of KLF-1 would affect its function in the intestine. KLF-1 ubiquitylation might cause a change in subcellular localization, so that KLF-1 is not available for proteasome-dependent degradation. However, we did not observe an obvious change in the cytoplasmic and nuclear localization of KLF-1 when expressed with WT WWP-1 or the $\mathrm{C} 762 \mathrm{~A}$ mutant, as determined by immunofluorescence in HEK293T cells (Supplementary Fig. 7). KLF-1 ubiquitylation by WWP-1 might alter the ability of KLF-1 to regulate gene transcription, for instance, by regulating KLF-1 binding to transcriptional regulators or specific gene promoters ${ }^{26}$. At this time, target genes regulated by KLF-1 in the worm have not been identified. It is unlikely that KLF-1 mediates transcription of 
stress response genes, as knockdown of klf-1 did not confer sensitivity to various stresses, including mild heat stress, oxidative stress and ultraviolet (Supplementary Fig. 8 and Supplementary Table 3).

Previous studies have reported that loss of $k l f-1$ results in increased fat storage in the intestine and cell death in the germline $^{3}$. Recent research has shown direct links between lipid metabolism, germline control and longevity ${ }^{27,28}$. Defects in fat metabolism and/or germline maintenance may contribute to the suppression of DR-induced longevity with loss of $k l f-1$. Indeed, worms undergoing DR have reduced intestinal fat stores, and loss of $k l f-1$ causes an increase of fat storage in the intestine ${ }^{3}$. It is believed that most of the life-extending benefits of DR are a consequence of its ability to reduce fat stores. In mice, long-term DR results in a shifted transcriptional phenotype reflecting energy metabolism activation in adipose tissue ${ }^{29}$. It is intriguing to speculate that klf-1 may regulate lipid status in the worm, which in turn affects aging, especially given that the C. elegans intestine is the major organ involved in lipid metabolism and is an important target organ in mediating lifespan extension ${ }^{30}$. Indeed, several mammalian KLF family members have been identified to be key players in the transcription of genes that control adipogenesis, lipogenesis and obesity ${ }^{2}$. Possibly, klf-1 exerts its role in the intestine via transcriptional regulation of genes modulating the activity of signalling pathways that control fat metabolism. Genes directly involved in lipid metabolism have been mapped to several signalling pathways that affect longevity including $\mathrm{DR}^{31}$. However, the exact role of lipids in modulation of aging remains unclear. Gene expression studies of worms with intestine-specific knockdown of WWP-1 and/or KLF-1 could help clarify the role of WWP-1 and KLF-1 in the DR pathway.

Perhaps C. elegans $k l f-1$, like pha-4, feeds into the target of rapamycin pathway to regulate longevity ${ }^{32}$. Indeed, we do find a genetic interaction between pha-4 and $k l f-1$ in regulating DRinduced longevity. There are numerous examples of Forkhead transcription factors regulating expression of KLFs and vice versa. For example, Drosophila forkhead binds to the Krüppel gene promoter and upregulates Krüppel gene expression ${ }^{33}$ and mammalian FOXO1 regulates KLF2, KLF4 and KLF6 expression $^{34-36}$. In addition, KLF4 has been shown to negatively regulate expression of FOXM1 (ref. 37) and ubiquitylated KLF10 upregulates FOXP3 expression ${ }^{11}$. Further dissection of the transcription network involving KLF-1 and PHA-4 would be critical in advancing our understanding of how DR enhances longevity.

\section{Methods}

C. elegans strains and methods. Nematodes were handled using standard methods ${ }^{38}$. WWP-1-overexpressing transgenic animals (Pwwp-1::green fluorescent protein::WWP-1) and the control line (Pwwp-1::green fluorescent protein) have been previously described ${ }^{13}$.

Generation of transgenic lines. Extrachromosomal arrays carrying transgenic strains were generated using standard microinjection methods ${ }^{39}$. For generation of N2 animals overexpressing klf-1, Pgly-19::KLF-1::tdTomato plasmid DNA was injected with the pRF4 (rol-6) construct. N2 animals injected with pRF4 were used as a control. For generation of eat-2(ad1116);sid-1(qt9) mutant animals with restored tissue-specific expression of SID-1, Pgly-19::SID-1 plasmid DNA was injected with Pgly-19::tdTomato plasmid DNA (for expression in intestinal tissue) or Pmyo-3::SID-1 plasmid DNA was injected with Pmyo-3::tdTomato (for expression in muscle tissue). Individual transgenic $\mathrm{F}_{1}$ progeny were selected on the basis of roller phenotype or tdTomato expression. Transgenic $F_{2}$ animals were isolated to establish independent lines.

Lifespan analysis. For lifespan analyses, synchronized populations of eggs were hatched and allowed to develop to the L4 stage. Once in the L4 stage, worms were transferred to new plates. The first day of adulthood is day 1 in survival curves. Worms were transferred to new plates every $24 \mathrm{~h}$ during the first six days of adulthood to eliminate confounding progeny. Animals were scored as alive, dead or censored every 2-3 days. Worms were scored as dead if they did not respond to repeated prods with a platinum pick. Animals that crawled off the plate, exploded or bagged were censored at the time of event. All lifespans were

performed at $20^{\circ} \mathrm{C}$ unless noted. Lifespan curves represent combined data from multiple independent experiments. eat-2(ad1116);CF512 larvae were plated at the restrictive temperature $\left(25^{\circ} \mathrm{C}\right)$ at day 1 of adulthood and then moved to and maintained at $20^{\circ} \mathrm{C}$ for the remainder of the lifespan. For bacterial dilution liquid culture lifespans, synchronized populations of eggs were hatched and grown at $20^{\circ} \mathrm{C}$ on nematode growth (NG) agar plates containing OP50 Escherichia coli until the L4 larval stage when they were transferred to plates of OP50 containing $100 \mu \mathrm{g} \mathrm{ml}^{-1}$ FUdR (to block worm reproduction). At day 1 of adulthood, worms were transferred into liquid culture containing OP50 bacteria at a concentration of

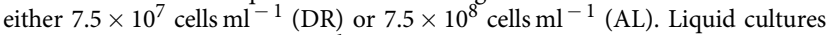
contained FUdR at $100 \mu \mathrm{g} \mathrm{ml}^{-1}$ for the first 4 days. Animals were transferred to new liquid cultures every 3-4 days and were scored as alive, dead or censored at the time of transfer. For bacterial dilution liquid cultures with RNAi knockdown, synchronized populations of eggs were hatched and grown on NG-carb agar plates containing bacteria expressing $k l f-1$ dsRNA, $w w p-1$ dsRNA or vector control to D1 adults when they were transferred to plates containing dsRNA-expressing bacteria and $100 \mu \mathrm{g} \mathrm{ml}^{-1} \mathrm{FUdR}$. At day 3 of adulthood, worms were transferred to liquid cultures containing OP50 bacteria at either DR or AL concentrations.

Statistical analysis. JMP8 software was used to determine means and percentiles $P$ values were calculated using the log-rank (Mantel-Cox method). $P$ values were calculated for individual experiments, each consisting of control and experimental animals examined at the same time. $P$ values were also calculated for combined data from multiple identical experiments.

RNAi treatment. RNAi-treated strains were fed E. coli (HT115) containing an empty vector or E. coli expressing dsRNA against the gene of interest. Unless indicated, worms were fed bacteria expressing dsRNA from hatching (L1). The wwp-1, pha-4, klf-2, ama-1, let-23, sek-1, mkk-4, rnt-1, ceh-6, lin-12 and hgrs-1 RNAi clones were from Julie Ahringer's RNAi library ${ }^{40}$. RNAi clones against $k l f-1$, $k l f-3$ and jun-1 were from Marc Vidal's RNAi library ${ }^{41}$.

Pumping rates. Pumping rates were determined by counting pumps of the terminal pharyngeal bulb for 1 min intervals. For each condition, worms were treated with bacteria expressing dsRNA since hatching. The pumping rates of 10 D1 adult worms per condition were measured and averaged.

Stress assays. For ultraviolet irradiation assays, worms were hatched and grown on specific RNAi-treated plates. At the L4 stage of development $100 \mu \mathrm{g} \mathrm{ml}^{-1}$ FUdR was added to the plates. D1 adults were transferred to plates without food and exposed to $1,200 \mathrm{~J} \mathrm{~m}^{-2}$ ultraviolet radiation using a Stratalinker UV Crosslinker (Stratagene). Worms were transferred back to fresh plates seeded with appropriate RNAi treatments and scored daily for viability. For paraquat (methyl viologen, Sigma) assays, worms were submerged in $50 \mu \mathrm{l}$ of $0.4 \mathrm{M}$ paraquat dissolved in S-basal buffer at day 5 of adulthood, after reproduction had ceased. Death was determined on an hourly basis by the lack of movement after prodding with a platinum wire. All assays were performed at $20^{\circ} \mathrm{C}$.

Plasmids and cell transfection. To generate N-terminally-tagged HA-WWP-1, $w w p-1$ (WT and C762A mutant) were subcloned into the pcDNA3 mammalian expression plasmid that had HA sequence inserted into the polylinker region. klf-1 cDNA cloned into pDONR201 was obtained from Open Biosystems. $k l f-1$ was subcloned into the pCMV-3Tag-1 vector (Agilent Technologies) to generate $\mathrm{N}$-terminally-tagged Flag $_{3}$-KLF-1 for mammalian expression. The ubiquitin construct used, which expresses eight tandem copies of HA-tagged ubiquitin under the control of the CMV promoter, has been previously described ${ }^{42}$. HEK293T cells were transfected with expression plasmids using polyethylenimine (Sigma) and collected $30 \mathrm{~h}$ later. Where indicated, $1 \mu \mathrm{M}$ bortezomib (Selleck Chemicals) was added to cells $24 \mathrm{~h}$ after transfection. Samples were harvested $6 \mathrm{~h}$ after bortezomib treatment.

Immunoprecipitation and immunoblot assays. For immunoprecipitation assays, cell pellets were resuspended in $1 \% \mathrm{NP} 40$ lysis buffer $(50 \mathrm{mM}$ Tris- $\mathrm{HCl}(\mathrm{pH} 8.0)$, $150 \mathrm{mM} \mathrm{NaCl}, 1 \% \mathrm{NP} 40$; the following was added to the buffer before lysis: $1 \mathrm{mM}$ dithiothreitol, $0.1 \mathrm{mM} \mathrm{Na} \mathrm{VO}_{4}, 0.1 \mathrm{mM}$ phenylmethylsulphonyl fluoride,

$1 \mu \mathrm{g} \mathrm{ml}^{-1}$ aprotinin). FLAG-KLF-1 was immunoprecipitated using anti-FLAG M2 affinity gel (Sigma). Immunoprecipitates were washed four times with lysis buffer and subjected to SDS-polyacrylamide gel electrophoresis. Gels were transferred to polyvinylidene difluoride membranes and probed with a monoclonal HA epitope tag antibody (ascites fluid from 12CA5 hybridoma cells) at a dilution of $1 / 10,000$ to detect HA-WWP-1 and a monoclonal FLAG M2 epitope tag antibody (Sigma) at a dilution of $1 / 1,000$ to detect FLAG-KLF-1. 
Immunofluorescence assays. For immunofluorescence assays, HEK293T cells were plated on glass coverslips and transfected with the indicated plasmids. Cells were then fixed with $4 \%$ paraformaldehyde and stained with a monoclonal antiFLAG antibody (M2) (Sigma) at a dilution of $1 / 1,000 \mu \mathrm{l}$ to detect KLF-1 expression, followed by incubation with a mouse secondary antibody (IgG) conjugated to Alexa Fluor 488 (Molecular Probes) at a dilution of 1/500. Cells were counterstained with Hoechst to identify all nuclei before mounting on slides.

Microscopy. For fluorescence microscopy of transgenic worms, worms were anesthetized with $50 \mathrm{mM}$ sodium azide on NG agar plates and were analysed using a Leica S6E dissecting microsope. Immunostained 293T cells were analysed using a Leica 6000B digital microscope. Images were acquired using Leica FW4000 and AF6000 software.

In vivo ubiquitylation assay. Transfected cells were washed two times with phosphate-buffered saline containing $2 \mathrm{mM} \mathrm{N}$-ethyl maleimide (to block the activity of isopeptidases and deubiquitylating enzymes). Cells were immediately lysed on the plate with preheated $\left(95^{\circ} \mathrm{C}\right) 2 \%$ SDS lysis buffer $(50 \mathrm{mM}$ Tris-HCl ( $\mathrm{pH} 7.4$ ), $150 \mathrm{mM} \mathrm{NaCl}, 2 \%$ SDS; the following was added to the buffer immediately before lysis: $1 \mathrm{mM}$ dithiothreitol, $0.1 \mathrm{mM} \mathrm{Na}_{3} \mathrm{VO}_{4}, 0.1 \mathrm{mM}$ phenylmethylsulphonyl fluoride, $1 \mathrm{~g} \mathrm{ml}^{-1}$ aprotinin and $4 \mathrm{mM} \mathrm{N}$-ethyl maleimide). Lysates were boiled for $10 \mathrm{~min}$. Denatured samples were then diluted in $1 \%$ Triton $\mathrm{X}-100$ lysis buffer (50 mM Tris- $\mathrm{HCl}$ ( $\mathrm{pH} 7.4), 150 \mathrm{mM} \mathrm{NaCl}, 1 \%$ Triton X-100; the following was added to the buffer immediately before lysis: $1 \mathrm{mM}$ dithiothreitol, $0.1 \mathrm{mM} \mathrm{Na} \mathrm{VO}_{4}, 0.1 \mathrm{mM}$ phenylmethylsulphonyl fluoride, $1 \mu \mathrm{g} \mathrm{ml}{ }^{-1}$ aprotinin and $2 \mathrm{mM} N$-ethyl maleimide) so that the final volume of SDS was reduced to $0.1 \%$. Cells were left on ice for $30 \mathrm{~min}$ and insoluble material was removed by centrifugation. Anti-FLAG M2 affinity gel (Sigma) was added to the lysates and incubated overnight at $4{ }^{\circ} \mathrm{C}$. Immunoprecipitates were washed four times with $1 \%$ Triton X-100 lysis buffer and subjected to SDS-polyacrylamide gel electrophoresis. Gels were transferred to polyvinylidene difluoride membranes and probed with the mouse monoclonal multi-ubiquitin FK1 antibody (MBL), which recognizes K6-, K11-, K27-, K33-, K29-, K48- and K63-linked ubiquitin chains but not monoubiquitin linkages. After stripping, the membrane was then probed with the mouse monoclonal P4D1 ubiquitin antibody (Santa Cruz) to detect total ubiquitin conjugates (polyubiquitin and monoubiquitin linkages). The membrane was then stripped again and probed with the rabbit polyclonal K48-specific ubiquitin antibody Apu2 (Millipore). The FK1, P4D1 and Apu2 antibodies were used at a dilution of $1 / 1,000$. Similar results were seen in an identical independent experiment where the membrane was first probed with the Apu2 antibody (data not shown).

Recombinant proteins and GST pull-down experiments. His-E1, GST-WWP-1 (WT and C762A mutant) and UBC-18 recombinant proteins have been previously described $^{13}$. The WW domain-containing region of WWP-1 (Amino acids 215-412) was cloned by PCR and subcloned into the pGEX6P1 bacterial expression vector to generate GST-WW. GST purifications were performed according to the manufacturer's instructions. $k l f-1$ and pha- 4 cDNAs were subcloned into the pCRII vector (Invitrogen) and used in the TnT Coupled Transcription/Translation Systems (Promega) to produce ${ }^{35} \mathrm{~S}$-methionine-labelled protein. For pull-down experiments, 5 or $10 \mu \mathrm{l}$ of ${ }^{35} \mathrm{~S}$-labelled lysates were incubated with $3.3 \mu \mathrm{g}$ of GST or GST fusion protein bound to glutathione beads in binding buffer $(50 \mathrm{mM}$ Tris- $\mathrm{HCl}$ (pH 8.0), $200 \mathrm{mM} \mathrm{NaCl}, 1 \mathrm{mM}$ EDTA, $1 \% \mathrm{NP} 40,10 \mathrm{mM} \mathrm{MgCl}_{2}$ and $1 \mathrm{mM}$ dithiothreitol) for $2 \mathrm{~h}$ at $4{ }^{\circ} \mathrm{C}$. Beads were washed four times with binding buffer and analysed by SDS-polyacrylamide gel electrophoresis. ${ }^{35}$ S-labelled proteins were identified by autoradiography.

In vitro ubiquitylation assay. The ubiquitylation reaction mix contained $20 \mu \mathrm{g}$ WT ubiquitin, ubiquitin mutants or methyl ubiquitin (Boston Biochem), $1 \mu \mathrm{M}$ ubiquitin aldehyde, $0.5 \mu \mathrm{g}$ His-E1, $0.2 \mu \mathrm{g}$ UBC-18, $0.25 \mu \mathrm{g}$ GST-WWP-1 (WT or C762A mutant) and $1 \mu \mathrm{l}$ in vitro-translated ${ }^{35} \mathrm{~S}$-labelled KLF-1 in $10 \mu \mathrm{l}$ reaction buffer ( $50 \mathrm{mM}$ Tris- $\mathrm{HCl}, \mathrm{pH} 7.5,5 \mathrm{mM} \mathrm{MgCl}_{2}, 1 \mathrm{mM}$ dithiothreitol and $5 \mathrm{mM}$ ATP). Following incubation at $30^{\circ} \mathrm{C}$ for $90 \mathrm{~min}$, ubiquitylation reactions were stopped with sample buffer and run on protein gels under denaturing conditions. Ubiquitylated KLF-1 forms were identified by autoradiography.

\section{References}

1. McConnell, B. B. \& Yang, V. W. Mammalian Krüppel-like factors in health and diseases. Physiol. Rev. 90, 1337-1381 (2010)

2. Brey, C. W., Nelder, M. P., Hailemariam, T., Gaugler, R. \& Hashmi, S. Krüppellike family of transcription factors: an emerging new frontier in fat biology. Int. J. Biol. Sci. 5, 622-636 (2009).

3. Hashmi, S. et al. A Krüppel-like factor in Caenorhabditis elegans with essential roles in fat regulation, cell death, and phagocytosis. DNA Cell Biol. 27, 545-551 (2008).

4. Zhang, J. et al. Regulation of lipoprotein assembly, secretion and fatty acid beta-oxidation by Kruppel-like transcription factor, klf-3. J. Mol. Biol. 425, 2641-2655 (2013)
5. Zhang, J. et al. Mutation in Caenorhabditis elegans Krüppel-like factor, KLF-3 results in fat accumulation and alters fatty acid composition. Exp. Cell Res. 315, 2568-2580 (2009).

6. Zhang, J. et al. Regulation of fat storage and reproduction by Krüppel-like transcription factor KLF3 and Fat-associated genes in Caenorhabditis elegans. J. Mol. Biol. 411, 537-553 (2011).

7. Plenefisch, J. D., Zhu, X. \& Hedgecock, E. M. Fragile skeletal muscle attachments in dystrophic mutants of Caenorhabditis elegans: isolation and characterization of the mua genes. Development 127, 1197-1207 (2000).

8. Conkright, M. D., Wani, M. A. \& Lingrel, J. B. Lung Krüppel-like factor contains an autoinhibitory domain that regulates its transcriptional activation by binding WWP1, an E3 ubiquitin ligase. J. Biol. Chem. 276, 29299-29306 (2001).

9. Chen, C. et al. Human Krüppel-like factor 5 is a target of the E3 ubiquitin ligase WWP1 for proteolysis in epithelial cells. J. Biol. Chem. 280, 41553-41561 (2005).

10. Zhang, X., Srinivasan, S. V. \& Lingrel, J. B. WWP1-dependent ubiquitination and degradation of the lung Kruppel-like factor KLF2. Biochem. Biophys. Res. Commun. 316, 139-148 (2004).

11. Venuprasad, K. et al. The E3 ubiquitin ligase itch regulates expression of transcription factor Foxp3 and airway inflammation by enhancing the function of transcription factor TIEG1. Nat. Immunol. 9, 245-253 (2008).

12. Peng, D. J. et al. Noncanonical K27-linked polyubiquitination of TIEG1 regulates Foxp3 expression and tumor growth. J. Immunol. 186, 5638-5647 (2011).

13. Carrano, A. C., Liu, Z., Dillin, A. \& Hunter, T. A conserved ubiquitination pathway determines longevity in response to diet restriction. Nature 460 , 396-399 (2009).

14. Panowski, S. H., Wolff, S., Aguilaniu, H., Durieux, J. \& Dillin, A. PHA-4/Foxa mediates diet-restriction-induced longevity of C. elegans. Nature 447, 550-555 (2007).

15. Libina, N., Berman, J. R. \& Kenyon, C. Tissue-specific activities of C. elegans DAF-16 in the regulation of lifespan. Cell 115, 489-502 (2003).

16. Winston, W. M., Molodowitch, C. \& Hunter, C. P. Systemic RNAi in C. elegans requires the putative transmembrane protein SID-1. Science 295, 2456-2459 (2002).

17. Xu, H. M. et al. WWP2, an E3 ubiquitin ligase that targets transcription factor Oct-4 for ubiquitination. J. Biol. Chem. 279, 23495-23503 (2004).

18. Jones, D. C. et al. Regulation of adult bone mass by the zinc finger adapter protein Schnurri-3. Science 312, 1223-1227 (2006).

19. Rossi, M. et al. The E3 ubiquitin ligase itch controls the protein stability of p63. Proc. Natl Acad. Sci. USA 103, 12753-12758 (2006).

20. Rossi, M. et al. The ubiquitin-protein ligase itch regulates p73 stability. EMBO J. 24, 836-848 (2005).

21. Gao, M. et al. Jun turnover is controlled through JNK-dependent phosphorylation of the E3 ligase itch. Science 306, 271-275 (2004).

22. Peschiaroli, A., Scialpi, F., Bernassola, F., El Sherbini el, S. \& Melino, G. The E3 ubiquitin ligase WWP1 regulates DeltaNp63-dependent transcription through Lys63 linkages. Biochem. Biophys. Res. Commun. 402, 425-430 (2010).

23. Zou, W. et al. The E3 ubiquitin ligase Wwp2 regulates craniofacial development through mono-ubiquitylation of Goosecoid. Nat. Cell. Biol. 13, 59-65 (2011).

24. Lee, T. L., Shyu, Y. C., Hsu, T. Y. \& Shen, C. K. Itch regulates p45/NF-E2 in vivo by Lys63-linked ubiquitination. Biochem. Biophys. Res. Commun. 375, 326-330 (2008).

25. Laine, A. \& Ronai, Z. Regulation of p53 localization and transcription by the HECT domain E3 ligase WWP1. Oncogene 26, 1477-1483 (2007).

26. Geng, F., Wenzel, S. \& Tansey, W. P. Ubiquitin and proteasomes in transcription. Annu. Rev. Biochem. 81, 177-201 (2012).

27. Ackerman, D. \& Gems, D. The mystery of C. elegans aging: an emerging role for fat: distant parallels between C. elegans aging and metabolic syndrome? Bioessays 8, 466-471 (2012).

28. Hansen, M., Flatt, T. \& Aguilaniu, H. Reproduction, fat metabolism, and life span: what is the connection? Cell Metab. 17, 10-19 (2013).

29. Higami, Y. et al. Energy restriction lowers the expression of genes linked to inflammation, the cytoskeleton, the extracellular matrix, and angiogenesis in mouse adipose tissue. J. Nutr. 136, 343-352 (2006).

30. Rera, M., Azizi, M. J. \& Walker, D. W. Organ-specific mediation of lifespan extension: more than a gut feeling? Ageing Res. Rev. 12, 436-444 (2013).

31. Hou, N. S. \& Taubert, S. Function and regulation of lipid biology in Caenorhabditis elegans aging. Front Physiol. 3, 143 (2012).

32. Sheaffer, K. L., Updike, D. L. \& Mango, S. E. The target of rapamycin pathway antagonizes pha-4/FoxA to control development and aging. Curr. Biol. 18, 1355-1364 (2008).

33. Kaufmann, E., Hoch, M. \& Jackle, H. The interaction of DNA with the DNA binding domain encoded by the Drosophila gene fork head. Eur. J. Biochem. 223, 329-337 (1994) 
34. Fabre, S. et al. FOXO1 regulates L-Selectin and a network of human T cell homing molecules downstream of phosphatidylinositol 3-kinase. J. Immunol. 181, 2980-2989 (2008).

35. Yusuf, I. et al. KLF4 is a FOXO target gene that suppresses B cell proliferation. Int. Immunol. 20, 671-681 (2008).

36. Sangodkar, J. et al. Targeting the FOXO1/KLF6 axis regulates EGFR signaling and treatment response. J. Clin. Invest. 122, 2637-2651 (2012).

37. Kong, X. et al. Dysregulated expression of FOXM1 Isoforms drives progression of pancreatic cancer. Cancer Res. 73, 3987-3996 (2013).

38. Brenner, S. The genetics of Caenorhabditis elegans. Genetics 77, 71-94 (1974).

39. Mello, C. C., Kramer, J. M., Stinchcomb, D. \& Ambros, V. Efficient gene transfer in C.elegans: extrachromosomal maintenance and integration of transforming sequences. Embo J. 10, 3959-3970 (1991).

40. Kamath, R. S. et al. Systematic functional analysis of the Caenorhabditis elegans genome using RNAi. Nature 421, 231-237 (2003).

41. Rual, J. F. et al. Toward improving Caenorhabditis elegans phenome mapping with an ORFeome-based RNAi library. Genome Res. 14, 2162-2168 (2004).

42. Treier, M., Staszewski, L. M. \& Bohmann, D. Ubiquitin-dependent c-Jun degradation in vivo is mediated by the delta domain. Cell 78, 787-798 (1994).

\section{Acknowledgements}

We thank members of the Hunter and Dillin laboratories for discussion and Zheng Liu, Huaiyu Sun and Mike French for their help. We thank Suzanne Wolff for generating the eat-2(ad1116);CF512 strain and Siler Panowski for generating the eat-2(ad1116);sid-

1(qt9) tissue-specific strains. Some strains were provided by the Caenorhabditis Genetics
Center, which is funded by the NIH National Center for Research Resources. This work was supported by USPHS grants CA14195, CA54418, CA80100, CA82683 and CA116402 (to T.H.), and NIDDK 070696 and NIA AG027463 grants, and the Ellison Medical and Glenn Medical Foundations (to A.D.). A.C.C. was supported by the Ellison Medical Foundation. T.H. is a Frank and Else Schilling American Cancer Society Professor and the Renato Dulbecco Chair in Cancer Research.

\section{Author contributions}

A.C.C designed and performed the experiments and analysed the data. A.D. and T.H. supervised the design and interpretation. The manuscript was written by A.C.C. and edited by A.D. and T.H. All authors discussed the results and commented on the manuscript.

\section{Additional information}

Supplementary Information accompanies this paper at http://www.nature.com/ naturecommunications

Competing financial interests: The authors declare no competing financial interests.

Reprints and permission information is available online at http://npg.nature.com/ reprintsandpermissions/

How to cite this article: Carrano, A. C. et al. A Krüppel-like factor downstream of the E3 ligase WWP-1 mediates dietary-restriction-induced longevity in Caenorhabditis elegans. Nat. Commun. 5:3772 doi: 10.1038/ncomms4772 (2014). 